\title{
Article \\ Gel-Forming of Self-Assembling Peptides Functionalized with Food Bioactive Motifs Modulate DPP-IV and ACE Inhibitory Activity in Human Intestinal Caco-2 Cells
}

\author{
Raffaele Pugliese $^{1, *(\mathbb{D})}$, Martina Bartolomei ${ }^{2} \mathbb{D}$, Carlotta Bollati ${ }^{2} \mathbb{D}$, Giovanna Boschin ${ }^{2}$, Anna Arnoldi ${ }^{2}$ (D) \\ and Carmen Lammi ${ }^{2, *(D)}$ \\ 1 NeMO Lab, ASST Grande Ospedale Metropolitano Niguarda, 20162 Milan, Italy \\ 2 Department of Pharmaceutical Sciences, University of Milan, 20133 Milan, Italy; \\ martina.bartolomei@unimi.it (M.B.); carlotta.bollati@unimi.it (C.B.); giovanna.boschin@unimi.it (G.B.); \\ anna.arnoldi@unimi.it (A.A.) \\ * Correspondence: raffaele.pugliese@nemolab.it (R.P.); carmen.lammi@unimi.it (C.L.)
}

\section{check for} updates

Citation: Pugliese, R.; Bartolomei, M.; Bollati, C.; Boschin, G.; Arnoldi, A.; Lammi, C. Gel-Forming of Self-Assembling Peptides Functionalized with Food Bioactive Motifs Modulate DPP-IV and ACE Inhibitory Activity in Human Intestinal Caco-2 Cells. Biomedicines 2022, 10, 330. https://doi.org/ $10.3390 /$ biomedicines 10020330

Academic Editor: Toshihiro Kita

Received: 29 December 2021

Accepted: 27 January 2022

Published: 31 January 2022

Publisher's Note: MDPI stays neutral with regard to jurisdictional claims in published maps and institutional affiliations.

Copyright: (C) 2022 by the authors. Licensee MDPI, Basel, Switzerland. This article is an open access article distributed under the terms and conditions of the Creative Commons Attribution (CC BY) license (https:// creativecommons.org/licenses/by/ $4.0 /)$.

\begin{abstract}
Food bioactive peptides are increasingly used for formulating food products, nutraceuticals, and functional food, since they are generally considered safe for human consumption and metabolic syndrome prevention. They are also becoming popular as sustainable sources of novel functional biomaterials such as hydrogels, edible nanonutraceuticals, delivery systems, and packing materials. However, such food peptides are mostly unstable, and degrade during food processing, or in a gastrointestinal environment, thus resulting in low bioavailability precluding their practical applications. Here, we decided to functionalize the well-known and characterized self-assembling peptide RADA16 with two synthetic analogues of food bioactive peptides deriving from the hydrolysis of soybean glycinin and lupin $\beta$-conglutin (namely IAVPTGVA and LTFPGSAED) for control of and improvement in their gel-forming nanostructures, biomechanics, and biological features. Extensive characterization was performed via Circular Dichroism (CD) spectroscopy, Fourier Transform Infrared spectroscopy (FT-IR), Thioflavin T (ThT) binding assay, rheological measurements, and Atomic Force Microscopy (AFM) analysis. Lastly, since self-assembling peptides (SAPs) can be co-assembled with diluent SAPs (without a bioactive epitope) as an approach to control the density of biological signals and therefore attain enhanced bioactivity, we investigated the effect of the co-assembly of RADA16 and functionalized food bioactive SAPs (dubbed cAP-Soy1 and cAP-Lup1) for the growth of Caco-2 human intestinal cells and contextually we characterized their biological activities as DPP-IV and ACE inhibitors, in order to demonstrate their potential use for the prevention of metabolic syndrome.
\end{abstract}

Keywords: self-assembling peptides; co-assembly; supramolecular hydrogels; nano-nutraceuticals; dipeptidyl peptidase IV; angiotensin converting enzyme; bioactivity; mechanical properties

\section{Introduction}

IAVPTGVA and LTFPGSAED (dubbed, respectively, Soy1 and Lup1) are two peptides derived from the hydrolysis of soybean glycinin and lupin $\beta$-conglutin, respectively. Both peptides display multifunctional behavior, since they demonstrated to act as in vitro HMGCoAR and DPP-IV inhibitors, respectively [1-4]. On human HepG2 cells, through the inhibition of HMGCoAR activity, they can modulate the cholesterol metabolism pathway leading to the upregulation of the LDLR protein levels, thus improved the ability of hepatic cells to uptake LDL from the extracellular environment with an in vitro cholesterollowering activity $[3,5]$. In addition, both Soy1 and Lup1 drop the in vitro and in situ human Caco-2 cells DPP-IV activity [1].

However, such food-derived peptides show low stability, bioavailability (due to their rapid degradation by active peptidases located on the intestinal microvilli), and a poor propensity to form functional biomaterials (i.e., hydrogels), thus precluding their practical 
applications. As we previously reported, one strategy to deal with these issues might be the use of well-designed and controlled self-assembled supramolecular systems, such as self-assembling peptides (SAPs) to be combined with the bioactive peptides in order to create hybrid systems, in which monomers are non-covalently bonded [6-9]. Indeed, when Soy1 and Lup1 were non-covalently entrapped into the RADA16 self-assembling peptide, the stability of both peptides was significantly improved as well as their capability of inhibiting DPP-IV and ACE, suggesting that this strategy could be a viable platform for targeting metabolic diseases [10].

Since SAPs are amenable to multi-functionalizations with bioactive motifs to intimately interact with cells, cytokines, tissues, and so on; we decided to functionalize the well-known and characterized RADA16 SAP with the synthetic analogues of Soy1 and Lup1 at the N-terminus, in order to create a food bioactive assembling peptide (dubbed fAP) with improved biological properties.

Extending the peptide sequence to the $\mathrm{C}$ or $\mathrm{N}$-terminus, either during the peptide synthesis or subsequently using click-chemistry, the biofunctionalization of the SAPs can be obtained. Furthermore, differently functionalized SAPs, sharing the same self-assembling sequence, can be mixed together, thus displaying multiple functional motifs [11]. Using this simple strategy, it is feasible to customize, in terms of bioactivity, an SAP-based hydrogel for each new biomedical application.

Herein, by using the advantage of SAP biofunctionalization, we synthetized two

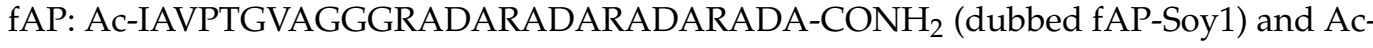
LTFPGSAEDGGGRADARADARADARADA-CONH 2 (dubbed fAP-Lup1) (Figure 1A). The N-terminus of both peptides was acetylated to increase their stability when in solution [12]; instead, a three-glycines spacer was used to avoid interference between the food bioactive motifs and the self-assembling backbone [13]. The secondary structures, biomechanics, and nanostructure morphologies of fAP hydrogels were extensively characterized by Fourier transform infrared spectroscopy (FT-IR), circular dichroism (CD), Thioflavin T (ThT) spectroscopy assay, oscillatory stress rheology, and atomic force microscopy (AFM). Lastly, since SAPs can be co-assembled with diluent SAPs (without a bioactive epitope) as an approach to control the density of biological signals [14] and therefore attain enhanced bioactivity in cases where steric effects play an important role in signaling [15], we investigated the effect of the co-assembly of RADA16 and fAPs (dubbed cAP-Soy1 and cAP-Lup1) for the growth of Caco-2 human intestinal cells and contextually we characterized their biological activities as DPP-IV and ACE inhibitors, in order to demonstrate their potential use for the prevention of metabolic syndrome.

A

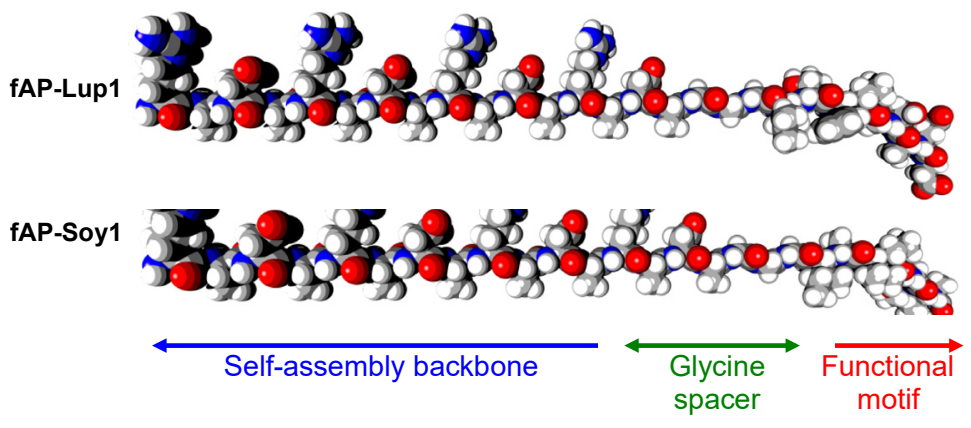

B

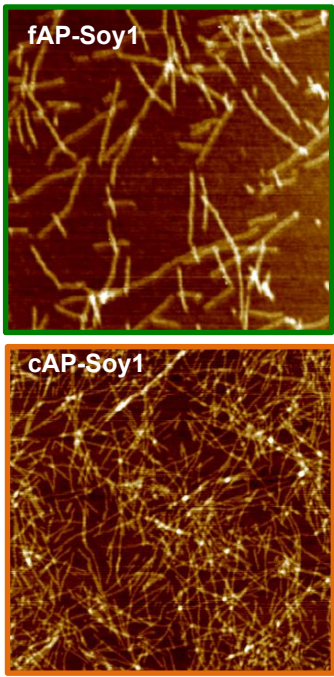

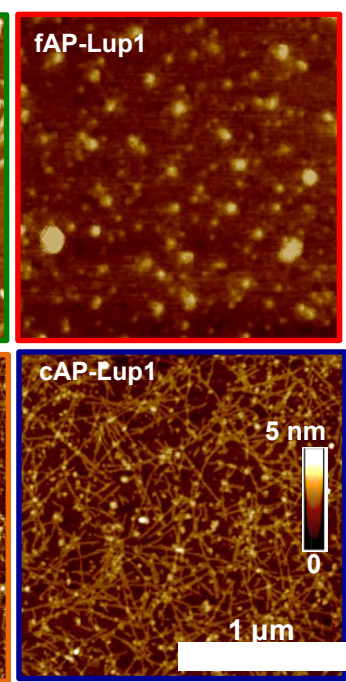

Figure 1. (A) Molecular graphics representation of fAP-Lup1 and fAP-Soy1 sequences. (B) Atomic force microscopy images of fAP-Soy1, fAP-Lup1, and co-assembled fAPs with a pure RADA16. 
This study may facilitate the development of naturally occurring food peptides as functional biomaterials with improved stability, bioavailability, and broaden their applications for metabolic syndrome prevention, food chemistry, coating for packaging, and beyond.

\section{Materials and Methods}

\subsection{Chemicals}

Dulbecco's modified Eagle's medium (DMEM), fetal bovine serum (FBS), phosphate buffered saline (PBS), penicillin/streptomycin, and 96-well plates were purchased from Euroclone (Milan, Italy). Gly-Pro-amido-4-methylcoumarin hydrobromide (Gly-Pro-AMC) and MTT [3-(4,5-dimethylthiazol-2-yl)-2,5-diphenyltetrazolium bromide] were from SigmaAldrich (St. Louis, MO, USA). Angiotensin I Converting Enzyme Activity (ACE1) Assay Kit came from BioVision (Milpitas, CA, USA).

\subsection{Peptide Preparation}

The synthetic analogues peptides were purchased from GenScript Biotech Corporation (Piscataway, NJ, USA). The purity of lyophilized peptides (>95\%) was tested using binary HPLC and LCMS mass spectrometry (Agilent 6520, Santa Clara, CA, USA) (Figure S1). Lyophilized peptides were dissolved at $1 \%(\mathrm{w} / \mathrm{v})$ in distilled water $\left(\mathrm{GIBCO}^{\circledR}\right.$ Thermo Fisher Scientific, Waltham, MA, USA), and then stored at $+4{ }^{\circ} \mathrm{C}$ overnight before use. For hydrogel preparation, 1X Dulbecco's Phosphate Buffered Saline (DPBS) $\left(\mathrm{Ca}^{2+} / \mathrm{Mg}^{2+}\right.$-free) was used, and the peptide solution was vigorously stirred for $5 \mathrm{~min}$.

\subsection{Thioflavin $T$ (ThT) Spectroscopy Assay}

ThT analysis of peptides was performed to assess the presence of amyloidogenic fibril structures. Peptide samples $(40 \mu \mathrm{M})$ were mixed with the ThT solution $(20 \mu \mathrm{M})$ and stirred for $4 \mathrm{~min}$, as previously reported [16]. The fluorescence intensity of ThT was checked through a Synergy plate reader (Biotek, Bad Friedrichshall, Germany) using $\lambda_{\mathrm{ex}}=440 \mathrm{~nm}$ ( $5 \mathrm{~nm}$ bandpass) and $\lambda_{\mathrm{em}}=482 \mathrm{~nm}$ (10 nm bandpass), over $60 \mathrm{~s}$ at $25^{\circ} \mathrm{C}$. Measurements were normalized over ThT-alone fluorescence and processed with OriginLab ${ }^{\mathrm{TM}} 8$ software.

\subsection{Fourier Transform Infrared Spectroscopy (FT-IR)}

The FT-IR spectra of peptides were obtained using a PerkinElmer Spectrum 100 spectrometer. Twenty acquisitions were recorded for each spectrum; the scan conditions were as follows: $4 \mathrm{~cm}^{-1}$ spectrum resolution, $25 \mathrm{kHz}$ scan speed, 1000-scan coaddition, and triangular apodization. All obtained spectra were reported after ATR correction, smoothing, and automatic baseline correction using OriginLab ${ }^{\mathrm{TM}} 8$ software.

\subsection{Circular Dichroism Spectroscopy (CD)}

The CD spectra (180-300 nm) of peptides were recorded on a Jasco J-815 (Jasco Corp., Tokyo, Japan) spectropolarimeter using a $0.1 \mathrm{~mm}$ quartz cuvette, as previously reported [17]. All scans were carried out with a scan speed of $50 \mathrm{~nm} / \mathrm{min}$, a bandwidth of $1 \mathrm{~nm}$, and time-response of $2 \mathrm{~s}$. A reference spectrum of distilled water was recorded and subtracted from each spectrum. All obtained spectra were reported using OriginLab ${ }^{\mathrm{TM}} 8$ software.

\subsection{Mechanical Testing}

To evaluate the storage $\left(G^{\prime}\right)$ and loss $\left(G^{\prime \prime}\right)$ moduli of assembled peptides $(1 \% \mathrm{w} / \mathrm{v})$, frequency sweep experiments were recorded as a function of angular frequency $(0.1-100 \mathrm{~Hz})$ at a fixed strain of $1 \%$, by using a stress/rate-controlled Rheometer (TA Instruments) equipped with a parallel plate geometry (acrylic diameter $20 \mathrm{~mm}$; gap $34 \mu \mathrm{m}$ ).

\subsection{Atomic Force Microscopy (AFM)}

AFM measurements were captured in tapping mode using a Tosca system (Anton Paar, Graz, Austria) using single-beam silicon cantilever probes. Peptides were dissolved in distilled water at a final concentration of $0.01 \% \mathrm{w} / \mathrm{v}$ and sonicated for $30 \mathrm{~min}$, the same 
day as imaging. Then, $5 \mu \mathrm{L}$ of peptide solution were deposited for $5 \mathrm{~min}$ on freshly cleaved mica, and distilled water was used to remove peptides loosely bound to the mica.

\subsection{Cell Culture}

Caco- 2 cells (INSERM, Paris, France), were sub-cultured at $50 \%$ density $\left(37^{\circ} \mathrm{C}, 90 \%\right.$ air, $10 \% \mathrm{CO}_{2}$ atmosphere) in Dulbecco Minimum Essential Medium (DMEM) containing $25 \mathrm{mM}$ glucose, $3.7 \mathrm{~g} / \mathrm{L} \mathrm{NaHCO}_{3}, 4 \mathrm{mM}$ stable L-glutamine, $1 \%$ nonessential amino acids, $100 \mathrm{U} / \mathrm{L}$ penicillin, $100 \mu \mathrm{g} / \mathrm{L}$ streptomycin (complete medium), supplemented with $10 \%$ heat-inactivated fetal bovine serum (FBS Hyclone Laboratories, Logan, UT, USA).

\subsection{In Situ DPPIV Activity Assay}

For 2D cell culture Caco-2 cells were seeded on the surface of cAP-Soy1 and cAP-Lup1 $(10$ and $100 \mu \mathrm{M})$ hydrogels at the density of $5 \times 10^{4} /$ well in a black 96-well plate with a clear bottom. The day after, the spent media was removed, cells were washed with $100 \mu \mathrm{L}$ of DPBS $\left(\mathrm{Ca}^{2+} / \mathrm{Mg}^{2+}\right.$ free), and $50 \mu \mathrm{L}$ of DPPIV substrate at the concentration of $50.0 \mu \mathrm{M}$ in DPBS $\left(\mathrm{Ca}^{2+} / \mathrm{Mg}^{2+}\right.$ free) were added in each well. Fluorescence signals using $\lambda_{\mathrm{ex}}=350 \mathrm{~nm}$ and $\lambda_{\mathrm{em}}=450 \mathrm{~nm}$ were measured using the Synergy H1 from Biotek (Bad Friedrichshall, Germany) every 1 min over $10 \mathrm{~min}$.

\subsection{In Situ ACE Activity Assay}

For the experiments, Caco- 2 cells at the density of $5 \times 10^{4}$ cells/well were seeded on cAP-Soy1 and cAP-Lup1 (10 and $100 \mu \mathrm{M})$ hydrogels in a 96-well plate and incubated for $24 \mathrm{~h}$ at $37^{\circ} \mathrm{C}$. The following day, the spent media was removed and $2 \mathrm{D}$ cell cultures were scraped in $50 \mu \mathrm{L} /$ well of ice-cold ACE1 lysis buffer and centrifuged at $4{ }^{\circ} \mathrm{C}, 13,300 \times g$, for $15 \mathrm{~min}$, and the supernatant was recovered and transferred into a new ice-cold tube. After quantifying the proteins using the Bradford method, $2 \mu \mathrm{g}$ of total protein (the equivalent of $2 \mu \mathrm{L}$ ) were added to $18 \mu \mathrm{L}$ of ACE1 lysis buffer in each well. Subsequently, $20 \mu \mathrm{L}$ of $4 \%$ ACE1 substrate (in assay buffer) were added and the fluorescence was measured in kinetic mode over $10 \mathrm{~min}$ at $37^{\circ} \mathrm{C}$ using $\lambda_{\mathrm{ex}}=330 \mathrm{~nm}$ and $\lambda_{\mathrm{em}}=430$. As a background control, $20 \mu \mathrm{L}$ of ACE1 lysis buffer in $20 \mu \mathrm{L}$ of ACE1 assay buffer were used.

\subsection{MTT Assay}

To assess the viability of Caco-2 cells seeded on the cAP-Soy1 and cAP-Lup1 (100 $\mu \mathrm{M})$ hydrogels, 3-(4,5-dimethylthiazol-2-yl)-2,5-diphenyltetrazolium bromide (MTT) assay was used. After $72 \mathrm{~h}$ of cell seeding, cells were incubated with $100 \mu \mathrm{L} /$ well of MTT reagent for $2 \mathrm{~h}$ at $37{ }^{\circ} \mathrm{C}$ in $5 \% \mathrm{CO}_{2}$. Afterwards, $0.5 \mathrm{mg} / \mathrm{mL}$ solution was removed and $100 \mu \mathrm{L} /$ well of the lysis buffer ( $8 \mathrm{mM} \mathrm{HCl}+0.5 \% \mathrm{NP}-40$ in DMSO) were added. The absorbance of each sample was measured at $575 \mathrm{~nm}$ using the Synergy H1 fluorescence plate reader (Biotek, Bad Friedrichshall, Germany).

\subsection{In Vitro Evaluation of DPP-IV and ACE Inhibitory Activity}

Peptide IAVP (1 mg/mL) was tested as previously described $[4,18,19]$ evaluating its DPP-IV and ACE inhibitory activity, respectively. For the evaluation of in vitro ACE inhibitory activity, a biochemical assay was used (further details are reported in supplementary materials).

\subsection{Statistical Analysis}

The entire data set was checked for normal distribution by $\mathrm{D}^{\prime}$ Agostino and Pearson test. Since they were all normally disturbed with $p$-values $<0.05$, we proceeded with statistical analyses by one-way ANOVA followed by Tukey's post-hoc tests using Graphpad Prism 9 (Graphpad, La Jolla, CA, USA). Values were expressed as means \pm s.d. of three independent experiments and each experiment was performed in triplicate; $p$-values $<0.05$ were considered to be significant. 


\section{Results}

\subsection{Supramolecular Organization of Peptide Nanostructures}

To gain insight into the nanostructure organization of fAPs, we used atomic force microscopy (AFM) morphological analysis (Figure 1B). Before the deposition on fresh mica, each peptide solution $(5 \mu \mathrm{L}$ ) was diluted to a final concentration of $0.01 \% \mathrm{w} / \mathrm{v}$ (see Section 2 for further details). The fAP-Soy1 yielded short single nanofibers with $10.02 \pm 1.8$ and $2.4 \pm 0.4$, respectively, in width and height, similarly to other reported functionalized RADA16 peptides [20-22]. In contrast, fAP-Lup1 did not form nanofibers, but instead led to the formation of sparse round aggregates; this is probably due to the presence of aromatic and aliphatic amino acids within the food bioactive motif. It was, therefore, co-assembled with a pure RADA16 that on its own self-assembles into long nanofibers (Figure S2) [23]. Co-assembly was achieved by mixing solutions of the fAP-Lup1 (1\% w/v) and RADA16 $(1 \% \mathrm{w} / \mathrm{v})$. After co-assembly, mixed solutions (namely cAP-Lup1) led to the formation of longer and much more numerous nanofibers if compared to individual fAP-Lup1 solution. The same was observed by mixing solutions of fAP-Soy1 and RADA16 (namely cAP-Soy1). Morphometrical analysis showed that fibers of cAPs ranged from $10 \pm 2.5$ to $11.65 \pm 1.6$ $\mathrm{nm}$ and $2.1 \pm 0.2$ to $2.5 \pm 0.6 \mathrm{~nm}$, respectively, in width and height.

Next, to investigate the supramolecular arrangement of fAP and cAP peptides, we performed Circular Dichroism (CD), Thioflavin-T (ThT)-binding assay, and Fourier Transform Infrared Spectroscopy (FT-IR).

To get an insight on the secondary structures of fAP and cAP assemblies, CD spectra were recorded in the far UV region of 180-300 nm (Figure 2A). All tested peptides showed a CD pattern comprising a negative peak at $215 \mathrm{~nm}$ and a positive peak at $195 \mathrm{~nm}$, characteristic of $\beta$-sheet secondary structures.
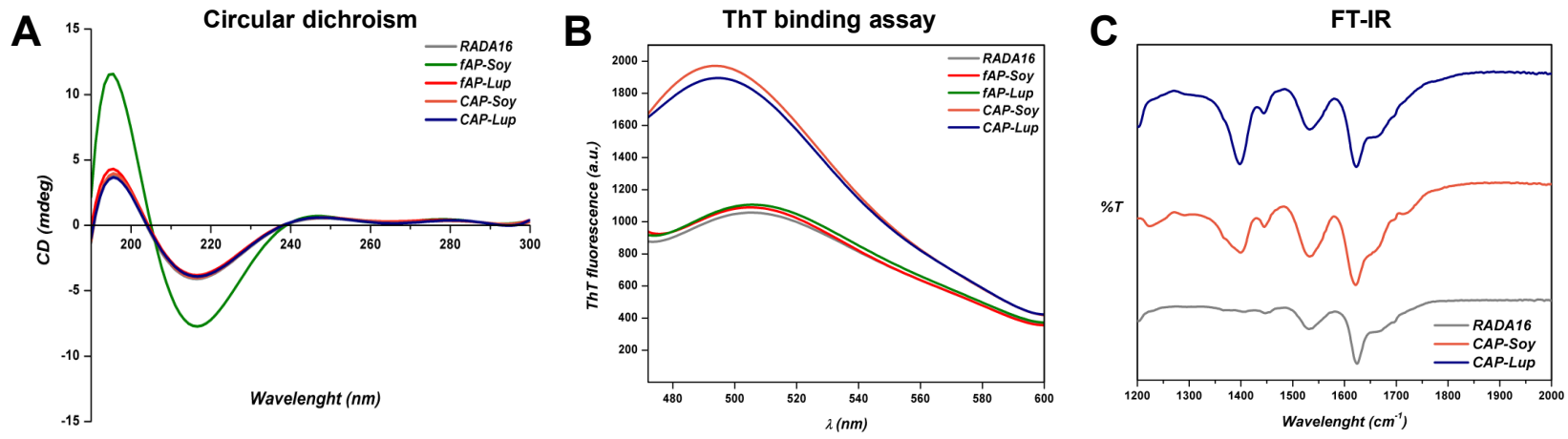

Figure 2. Supramolecular organization of fAPs and cAPs peptide solutions. (A) Circular Dichroism (CD) spectra of fAPs and cAPs suggesting the presence of $\beta$-sheet secondary structures; (B) ThTbinding assay of fAPs and cAPs hydrogels showing typical amyloid emission signals (centered at $\sim 500 \mathrm{~nm}$ ) that increase in co-assembled fAPs with a pure RADA16; (C) FT-IR spectra of fAPs and cAPs hydrogels with characteristic $\beta$-sheet peaks in the amide I and amide II regions.

Since the $\beta$-sheet structure is characteristic of amyloid-like fibers, typical of SAP molecules, we evaluated this behavior on fAP and cAP peptides using the thioflavin-T (ThT) binding assay [24] (Figure 2B). The fAP samples exhibited a fluorescence intensity peaked at $500 \mathrm{~nm}$ comparable to those of RADA16 wild type, ascribable to amyloidogenic structure emissions. Instead, co-assembled fAPs with a pure RADA16 showed high fluorescence levels, probably due to the conformational changes after mixing solutions, thus establishing their strong amyloid-like nature rich in $\beta$-sheet structures.

In order to further explore the secondary structure arrangement of both cAPs in solution, we carried out FT-IR spectroscopy tests (Figure 2C). FT-IR spectra of both cAP peptides exhibited a sharp amide I band at $1623 \mathrm{~cm}^{-1}$, indicating predominantly $\beta$-sheet features. The band at $1,532 \mathrm{~cm}^{-1}$ in the amide II region also confirmed the $\beta$-sheet aggregation of cAPs, in agreement with the RADA16 spectrum. In addition, both cAP-Soy1 and cAP-Lup1 
showed one broad peak at $1400 \mathrm{~cm}^{-1}$, which is ascribable to the ring stretching of proline and phenylalanine [25] present in the food bioactive motifs.

Altogether, these data confirmed the self-aggregation of all tested fAPs and cAPs into $\beta$-sheets secondary structures, with a characteristic amyloid-like fibers structure. Further, both CD and FT-IR data suggested that co-assembly by mixing solutions of the fAP-Lup1 and fAP-Soy1 with pure RADA16 does not impair the $\beta$-sheet formation but, on the contrary, promotes the formation of ordered $\beta$-sheet-based nanostructures.

\subsection{Mechanical Properties of Peptide Nanostructures}

After confirming the formation of stable $\beta$-sheet nanostructures of both fAP and cAPs hydrogels, biomechanical properties of each peptide solution were analyzed via shear-rate rheology (Figure 3). Usually, for $\beta$-sheet rich peptide nanofibers the growing presence of such structures leads to formation of an entangled fibrous network, that provides increased $G^{\prime}$ values [26]. Hence, the progression of $G^{\prime}$ and its comparison with $G^{\prime \prime}$ of all pre-assembled fAP and cAPs solutions were monitored via frequency sweep test $(0.1-100 \mathrm{~Hz}, 1 \%$ strain) to evaluate if they showed an elastic (i.e., $G^{\prime}>G^{\prime \prime}, \tan \delta<1$ ) or viscous (i.e., $G^{\prime}<G^{\prime \prime}, \tan \delta<1$ ) profile. Though $\mathrm{G}^{\prime}$ and $\mathrm{G}^{\prime \prime}$ remained relatively constant along the tested frequency range, only co-assembled peptide hydrogels displayed significantly increased $\mathrm{G}^{\prime}$ values (1379 Pa for CAP-Soy1 and 958 Pa for CAP-Lup1). Such values of $\mathrm{G}^{\prime}$ are probably due to higher organization of cAPs given by their $\beta$-sheets-like packed nature seen in ThT experiments, and by nanofiber morphologies shown in AFM analysis.

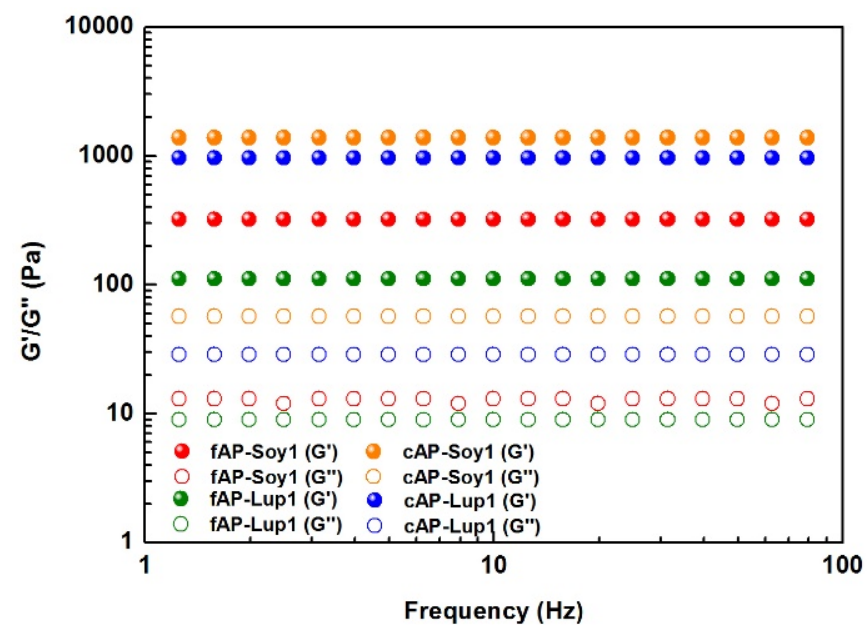

Figure 3. Rheological studies to evaluate the mechanical properties of fAP and cAP hydrogels. Frequency-dependent oscillatory rheology $(0.1-100 \mathrm{~Hz})$ of all assembled peptides $(1 \% \mathrm{w} / \mathrm{v})$ highlighted the typical profile of soft hydrogels, featuring a predominant solid-elastic behavior $\left(\mathrm{G}^{\prime}\right.$, full dots) as compared to the viscous component ( $G^{\prime \prime}$, empty dots).

\subsection{Biological Evaluation of Co-Assembling Peptide Nanostructures}

The potential of CAP-Soy1 and CAP-Lup1 as substrates for human intestinal Caco-2 cells was tested in vitro. A total of $5 \times 10^{4} /$ well Caco- 2 cells were seeded directly on the top surface of the cAP-Soy1 and cAP-Lup1 hydrogels (see Section 2 for further details). Cells were cultured for 2 and 3 days in vitro (DIV) in order to evaluate the ability of both peptides to act as cell culture scaffolds. As shown in Figure 4A, Caco-2 cells cultured over assembled cAP hydrogels showed spread and fusiform shapes without significant morphological changes compared to Caco-2 cells cultured on RADA16 hydrogel alone. No cytotoxicity effects were observed after 3 DIV by using MTT assay (Figure 4B). 

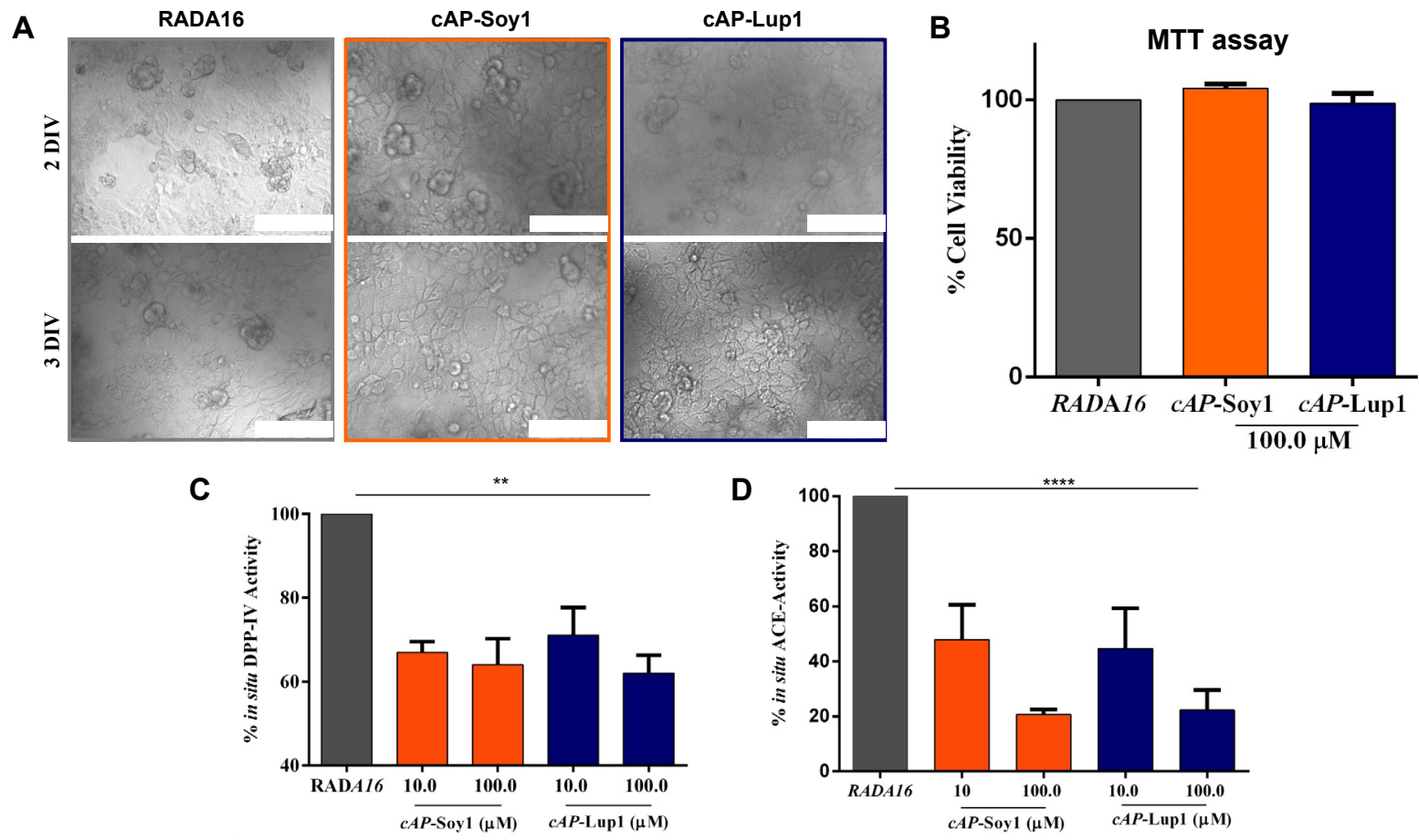

Figure 4. Biological evaluation of co-assembling peptide nanostructures. (A) Caco-2 cells cultured over assembled cAP hydrogels (scale bar $100 \mu \mathrm{m}$ ). (B) Effects of cAP-Soy1 and cAP-Lup1 on the Caco-2 cells viability. (C) Effects of cAP-Soy1 and cAP-Lup1 (10.0 and $100.0 \mu \mathrm{M})$ on DPP-IV and (D) ACE activities expressed by Caco-2 cells, respectively. Statistically analysis was carried out using one-way ANOVA followed by Tukey's post-hoc test. $\left.{ }^{* *}\right) p<0.01,\left({ }^{* * * *}\right) p<0.0001$. All data sets were statically different vs. RADA16, whereas no statistical significance was observed between cAP-Soy1 and cAP-Lup1 groups in both DPP-IV and ACE assays, respectively.

To assess the potential of cAP-Soy1 and CAP-Lup1 as substrates to modulate the DPP-IV and ACE, activity was tested in situ on human intestinal Caco- 2 cells. Notably, this cellular system is an interesting survey of membrane peptidases and among these, DPP-IV and ACE stand out [27]. Based on this consideration, our group has already developed, optimized, and validated dedicated cell-based assays in order to measure both DPP-IV [1] and ACE $[8,28]$ activity on Caco-2 cells.

In order to control the density of food biological signals and therefore attain enhanced bioactivity, thus avoiding the over-expression of the food bioactive motifs, we tested the cAP-based substrates at 10 and $100 \mu \mathrm{M}$, respectively.

We observed that CAP-Soy1 reduced the DPP-IV activity by $33.1 \pm 2.6 \%$ and $36.1 \pm 6.2 \%$ at 10 and $100 \mu \mathrm{M}$, respectively, whereas, cAP-Lup1 inhibited the same enzyme by $29.0 \pm 6.5 \%$ $(10 \mu \mathrm{M})$ and $38.0 \pm 4.3 \%(100 \mu \mathrm{M})$ (Figure $4 \mathrm{C})$. In addition, to assess the multifunctional properties of cAPs, ACE-inhibitory activity was investigated. Our findings indicate that cAP-Soy1 (10 and $100 \mu \mathrm{M})$ inhibits the ACE activity by $52.2 \pm 12.7 \%$ and $79.5 \pm 1.8 \%$, whereas cAP-Lup1 by $55.4 \pm 14.5 \%$ and $77.8 \pm 7.2 \%$ at 10 and $100 \mu \mathrm{M}$, respectively (Figure 4D).

Both the active motives, Soy1 and Lup1, are able to across the intestinal barrier and during the trans-epithelial transport both peptides are metabolized by the intestinal peptidases located on the apical side of the Caco- 2 cells, producing some break-down fragments. Notably, Soy1 is partially degraded in three small peptides AVPTGVA, IAVPT, and IAVP [29], whereas Lup1 is cleaved in two peptides TFPGSAED and LTFPG [28]. In detail, the analysis of the Soy1 sequence using BIOPEP suggests that both AVP and IA are known ACE inhibitor peptides from casein and soybean hydrolysates, respectively, whereas the sequences IA, AV, VP are known as DPP-IV inhibitors, respectively. Hence, experiments were performed for evaluating the ability of IAVP to inhibit both the ACE and 
DPP-IV activity, respectively. Results suggest that IAVP reduces the in vitro ACE activity by $20.1 \pm 0.5 \%$ at $1 \mathrm{mg} / \mathrm{mL}$, whereas it is totally ineffective on DPP-IV. Similarly, it has been demonstrated that LTFPG from Lup1 maintains its ACE inhibitory activity losing that against DPP-IV [28].

Based on these results and considering that both CAP-Soy1 and cAP-Lup1 are more active as ACE than DPP-IV inhibitors, it is feasible to conclude that when both functionalized cAPs are in contact with the Caco-2 cells, their intrinsic bioactivity is modulated by the cellular system to whom they come into contact. Indeed, their cleavable nature makes smart functionalized cAPs, which may expose a combination of active peptides confirming the cAPs dynamic multifunctional behavior.

\section{Discussion}

We described here a feasible strategy to control and boost up the biological activities of synthetic analogues of two food bioactive peptides derived from the hydrolysis of soybean glycinin and lupin $\beta$-conglutin. This was addressed by extending the RADA16 self-assembling peptide sequence at the $\mathrm{N}$-terminus with the two food-derived synthetic analogues. Adopting atomic force microscopy, spectroscopic and rheological techniques, we carried out a thorough investigation of their nanostructures, gelling properties, and biomechanical features. Furthermore, we showed that by co-assembling pure RADA16 with fAP-Soy1 and fAP-Lup1 (namely cAPs), as an approach to control the density of biological signals (i.e., IAVPTGVA and LTFPGSAED), it is possible to achieve greater bioactivity as DPP-IV and ACE inhibitors in Caco-2 cells.

We previously reported that the non-covalent encapsulation of Soy1 and Lup1 plain solutions within the pre-formed nano-fibrillar hydrogel of RADA16 provides not only higher resistance towards the proteases but also a higher bioavailability of such food bioactive peptides. Furthermore, when these peptides were entrapped within the RADA16 hydrogel, they were released slowly allowing their interaction with the DPP-IV and ACE catalytic sites, suggesting that this strategy could be a viable platform for targeting metabolic diseases.

However, the main limitation of this strategy is that we needed to encapsulate more than $100 \mu \mathrm{M}$ of both Soy1 and Lup1 to have a positive effect on DPP-IV and ACE, and then that the two peptides were released with different trends over time; in fact, while Lup1 was regularly delivered by the RADA16 hydrogel during the $360 \mathrm{~min}$ of controlled release tests, Soy1 was immediately released within 60-180 $\mathrm{min}$.

Instead, in this work by directly incorporating the synthetic analogues Lup1 and Soy1 sequences to the RADA16 sequence, through a three-glycines spacer to avoid interference between the bioactive motifs and the self-assembling backbone, we tried to bypass the previously reported constraints to improve their stability, bioavailability, and propensity to form bioactive functional hydrogels; as such, Lup1 and Soy1 remain localized and intrinsically exposed in the gelling nanostructures to interact intimately with the cells.

From a morphological point of view, we showed that fAP-Soy1 produces short single nanofibers, and conversely, fAP-Lup1 leads to the formation of round aggregates. Therefore, to ensure a correct nanofibers formation (similar to those of other functionalized RADA16 peptides), and to control the density of the food biological signals and thus obtain a greater bioactivity, avoiding their overexpression (which can be detrimental), we co-assembled fAP-Soy1 and fAP-Lup1 with a pure RADA16, thus leading to the formation of longer and much more numerous nanofibers. Further, CD and FT-IR data suggested that co-assembly by mixing solutions of the fAP-Lup1 and fAP-Soy1 with pure RADA16 does not impair the $\beta$-sheet formation (typical of RADA16-based hydrogels) but, on the contrary, promotes the formation of ordered $\beta$-sheet-based nanostructures. Moreover, rheological data revealed that co-assembled peptide hydrogels displayed significantly increased $G^{\prime}$ values, thus indicating the formation of stiffer and robust co-assembled functionalized hydrogels not only compared to the fAP-Soy1 and fAP-Lup1 samples, but also those previously reported in which Lup1 and Soy1 were entrapped within the pre-formed RADA16 hydrogel. 
Further, we have previously demonstrated that both plain solutions of bioactive motives displayed an interesting multifunctional behavior inhibiting the in vitro $\left(\mathrm{IC}_{50}\right.$ of 106 and $228 \mu \mathrm{M}$ ) and cellular ( $\mathrm{IC}_{50}$ of 223 and $208 \mu \mathrm{M}$ ) DPP-IV activity measured on purified enzymes and on that expressed by Caco- 2 cells, respectively [4]. Comparing the $\mathrm{IC}_{50}$ values, it appears clear that Soy1 is more active than Lup1 in the biochemical condition, whereas when the same peptides were tested in a cell-based environment, both peptides displayed similar DPP-IV inhibitory potencies. This difference was explained demonstrating that Soy1 is more suitable for the metabolic activity of peptidase expressed by the apical side of human intestinal Caco-2 cells than Lup1 [28]. Indeed, Soy1 (IAVPTGVA) is metabolized in three breakdown fragments (AVPTGVA, IAVP, and IAV) [29], whereas Lup1 (LTFPGSDAD) is degraded only in one metabolite (LTFPG) [28]. In addition, both peptides reduce the ACE activity expressed by Caco- 2 cells with a dose-response trend and comparable $\mathrm{IC}_{50}$ values, which are equal to 14.7 and $13.7 \mu \mathrm{M}$, respectively.

In light of these considerations, our results confirm the multifunctional behavior of both cAP-Soy1 and cAP-Lup1. Indeed, we demonstrated that both intrinsically bioactive hydrogels reduce the ACE and DPP-IV activities, respectively. In more detail, we tested both cAP-Soy1 and CAP-Lup1 at the fixed concentrations of 100 and $10 \mu \mathrm{M}$, since these concentrations represent a good compromise with the biological activity on both DPP-IV and ACE enzymes, respectively. In addition, also from a biological point of view the strategy presented in this work provides a useful and alternative system to the encapsulation of bioactive peptides within the RADA16 nanofibers for improving their stability and activity.

\section{Conclusions}

In conclusion, we synthesized and characterized a nanofiber-based SAP functionalized with two food bioactive peptides derived from the hydrolysis of soybean glycinin and lupin $\beta$-conglutin as a viable strategy for controlling and improving their nanostructures, biomechanics, as well as to control the density of food biological signals and therefore attain enhanced bioactivity.

The nanofiber morphology and multivalent presentation of IAVPTGVA and LTFPGSAED peptides as DPP-IV and ACE inhibitors in vitro on Caco-2 human intestinal cells could offer a future targeting strategy for the prevention of metabolic syndrome through their consumption as edible nanonutraceuticals.

Future studies will focus on the in vivo therapeutic effect of such nanonutraceuticals to inhibit DPP-IV and ACE and test their behavior to modulate the cholesterol metabolism pathway for targeting the metabolic diseases.

Supplementary Materials: The following are available online at https:/ /www.mdpi.com/article/10 .3390/biomedicines10020330/s1, Figure S1. HPLC and LCMS spectra of fAP-Soy1 and fAP-Lup1; Figure S2. Atomic force microscopy images of pure RADA16.

Author Contributions: Conceptualization, R.P. and C.L.; methodology, R.P. and C.L.; software, R.P., C.L.; validation, R.P., M.B., C.B., C.L.; investigation, R.P., M.B., C.B., G.B., A.A., C.L.; data curation, R.P. and C.L.; writing—original draft preparation, R.P., A.A., C.L.; writing—review and editing, R.P., A.A., C.L.; supervision, R.P. and C.L.; project administration, R.P. and C.L.; funding acquisition, R.P., A.A., C.L. All authors have read and agreed to the published version of the manuscript.

Funding: This research was in part funded by UNIMI, grant "linea 2".

Institutional Review Board Statement: Not applicable.

Informed Consent Statement: Not applicable.

Data Availability Statement: Data is contained within the article or supplementary material.

Conflicts of Interest: The authors declare no conflict of interest. 


\section{References}

1. Lammi, C.; Bollati, C.; Ferruzza, S.; Ranaldi, G.; Sambuy, Y.; Arnoldi, A. Soybean- and Lupin-Derived Peptides Inhibit DPP-IV Activity on In Situ Human Intestinal Caco-2 Cells and Ex Vivo Human Serum. Nutrients 2018, 10, 1082. [CrossRef] [PubMed]

2. Lammi, C.; Zanoni, C.; Arnoldi, A.; Vistoli, G. Two Peptides from Soy beta-Conglycinin Induce a Hypocholesterolemic Effect in HepG2 Cells by a Statin-Like Mechanism: Comparative in Vitro and in Silico Modeling Studies. J. Agric. Food Chem. 2015, 63, 7945-7951. [CrossRef] [PubMed]

3. Lammi, C.; Zanoni, C.; Arnoldi, A. Three Peptides from Soy Glycinin Modulate Glucose Metabolism in Human Hepatic HepG2 Cells. Int. J. Mol. Sci. 2015, 16, 27362-27370. [CrossRef] [PubMed]

4. Lammi, C.; Zanoni, C.; Arnoldi, A.; Vistoli, G. Peptides Derived from Soy and Lupin Protein as Dipeptidyl-Peptidase IV Inhibitors: In Vitro Biochemical Screening and in Silico Molecular Modeling Study. J. Agric. Food Chem. 2016, 64, 9601-9606. [CrossRef]

5. Zanoni, C.; Aiello, G.; Arnoldi, A.; Lammi, C. Investigations on the hypocholesterolaemic activity of LILPKHSDAD and LTFPGSAED, two peptides from lupin $\beta$-conglutin: Focus on LDLR and PCSK9 pathways. J. Funct. Foods 2017, 32, 1-8. [CrossRef]

6. Lammi, C.; Bollati, C.; Gelain, F.; Arnoldi, A.; Pugliese, R. Enhancement of the Stability and Anti-DPPIV Activity of Hempseed Hydrolysates Through Self-Assembling Peptide-Based Hydrogels. Front. Chem. 2018, 6, 670. [CrossRef]

7. Pugliese, R.; Bollati, C.; Gelain, F.; Arnoldi, A.; Lammi, C. A Supramolecular Approach to Develop New Soybean and Lupin Peptide Nanogels with Enhanced Dipeptidyl Peptidase IV (DPP-IV) Inhibitory Activity. J. Agric. Food Chem. 2019, 67, 3615-3623. [CrossRef]

8. Dellafiora, L.; Pugliese, R.; Bollati, C.; Gelain, F.; Galaverna, G.; Arnoldi, A.; Lammi, C. “Bottom-Up” Strategy for the Identification of Novel Soybean Peptides with Angiotensin-Converting Enzyme Inhibitory Activity. J. Agric. Food Chem. 2020, 68, 2082-2090. [CrossRef]

9. Pugliese, R.; Arnoldi, A.; Lammi, C. Nanostructure, Self-Assembly, Mechanical Properties, and Antioxidant Activity of a Lupin-Derived Peptide Hydrogel. Biomedicines 2021, 9, 294. [CrossRef]

10. Pugliese, R.; Gelain, F.; Arnoldi, A.; Lammi, C. Chemistry and Functional Roles of Food Protein Hydrogels. In Food Proteins and Peptides: Emerging Biofunctions, Food and Biomaterial Applications; Royal Society of Chemistry: Cambridge, UK, 2021.

11. Pugliese, R.; Fontana, F.; Marchini, A.; Gelain, F. Branched peptides integrate into self-assembled nanostructures and enhance biomechanics of peptidic hydrogels. Acta Biomater. 2018, 66, 258-271. [CrossRef]

12. Pugliese, R.; Gelain, F. Peptidic Biomaterials: From Self-Assembling to Regenerative Medicine. Trends Biotechnol. 2017, 35, 145-158. [CrossRef] [PubMed]

13. Taraballi, F.; Natalello, A.; Campione, M.; Villa, O.; Doglia, S.M.; Paleari, A.; Gelain, F. Glycine-spacers influence functional motifs exposure and self-assembling propensity of functionalized substrates tailored for neural stem cell cultures. Front. Neuroeng. 2010, 3, 1. [CrossRef] [PubMed]

14. Storrie, H.; Guler, M.O.; Abu-Amara, S.N.; Volberg, T.; Rao, M.; Geiger, B.; Stupp, S.I. Supramolecular crafting of cell adhesion. Biomaterials 2007, 28, 4608-4618. [CrossRef] [PubMed]

15. Boekhoven, J.; Stupp, S.I. 25th anniversary article: Supramolecular materials for regenerative medicine. Adv. Mater. 2014, 26, 1642-1659. [CrossRef]

16. Pugliese, R.; Gelain, F. Cross-Linked Self-Assembling Peptides and Their Post-Assembly Functionalization via One-Pot and In Situ Gelation System. Int. J. Mol. Sci. 2020, 21, 4261. [CrossRef]

17. Aiello, G.; Pugliese, R.; Rueller, L.; Bollati, C.; Bartolomei, M.; Li, Y.; Robert, J.; Arnoldi, A.; Lammi, C. Assessment of the Physicochemical and Conformational Changes of Ultrasound-Driven Proteins Extracted from Soybean Okara Byproduct. Foods 2021, 10, 562. [CrossRef]

18. Boschin, G.; Scigliuolo, G.M.; Resta, D.; Arnoldi, A. ACE-inhibitory activity of enzymatic protein hydrolysates from lupin and other legumes. Food Chem. 2014, 145, 34-40. [CrossRef]

19. Boschin, G.; Scigliuolo, G.M.; Resta, D.; Arnoldi, A. Optimization of the Enzymatic Hydrolysis of Lupin (Lupinus) Proteins for Producing ACE-Inhibitory Peptides. J. Agric. Food Chem. 2014, 62, 1846-1851. [CrossRef]

20. Wang, J.; Zheng, J.; Zheng, Q.; Wu, Y.; Wu, B.; Huang, S.; Fang, W.; Guo, X. FGL-functionalized self-assembling nanofiber hydrogel as a scaffold for spinal cord-derived neural stem cells. Mater. Sci Eng. C Mater. Biol. Appl. 2015, 46, 140-147. [CrossRef]

21. Wang, X.-M.; Lin, Q.; Horii, A. Screening of functionalized self-assembling peptide nanofiber scaffolds with angiogenic activity for endothelial cell growth. Prog. Nat. Sci. Mater. Int. 2011, 21, 111-116. [CrossRef]

22. Taraballi, F.; Campione, M.; Sassella, A.; Vescovi, A.; Paleari, A.; Hwang, W.; Gelain, F. Effect of functionalization on the self-assembling propensity of $\beta$-sheet forming peptides. Soft Matter 2009, 5, 660-668. [CrossRef]

23. Gelain, F.; Luo, Z.; Zhang, S. Self-Assembling Peptide EAK16 and RADA16 Nanofiber Scaffold Hydrogel. Chem. Rev. 2020, 120, 13434-13460. [CrossRef] [PubMed]

24. Xue, C.; Lin, T.Y.; Chang, D.; Guo, Z. Thioflavin T as an amyloid dye: Fibril quantification, optimal concentration and effect on aggregation. R. Soc. Open Sci. 2017, 4, 160696. [CrossRef] [PubMed]

25. Barth, A. The infrared absorption of amino acid side chains. Progress in Biophysics and Molecular Biology 2000, 74, 141-173. [CrossRef]

26. Yan, C.; Pochan, D.J. Rheological properties of peptide-based hydrogels for biomedical and other applications. Chem. Soc. Rev. 2010, 39, 3528-3540. [CrossRef] 
27. Howell, S.; Kenny, A.J.; Turner, A.J. A survey of membrane peptidases in two human colonic cell lines, Caco-2 and HT-29. Biochem. J. 1992, 284 Pt 2, 595-601. [CrossRef]

28. Lammi, C.; Aiello, G.; Dellafiora, L.; Bollati, C.; Boschin, G.; Ranaldi, G.; Ferruzza, S.; Sambuy, Y.; Galaverna, G.; Arnoldi, A. Assessment of the Multifunctional Behavior of Lupin Peptide P7 and Its Metabolite Using an Integrated Strategy. J. Agric. Food Chem. 2020, 68, 13179-13188. [CrossRef]

29. Aiello, G.; Ferruzza, S.; Ranaldi, G.; Sambuy, Y.; Arnoldi, A.; Vistoli, G.; Lammi, C. Behavior of three hypocholesterolemic peptides from soy protein in an intestinal model based on differentiated Caco-2 cell. J. Funct. Foods 2018, 45, 363-370. [CrossRef] 